\title{
Corrigendum
}

\section{iNKT cells require TSC1 for terminal maturation and effector lineage fate decisions}

Jinhong Wu, Jialong Yang, Kai Yang, Hongxia Wang, Balachandra Gorentla, Jinwook Shin, Yurong Qiu, Loretta G. Que, W. Michael Foster, Zhenwei Xia, Hongbo Chi, and Xiao-Ping Zhong

Original citation: J Clin Invest. 2014;124(4):1685-1698. https://doi.org/10.1172/JCI69780.

Citation for this corrigendum: J Clin Invest. 2018;128(4):1707-1708. https://doi.org/10.1172/JCI120531.

The Editors recently posted an Expression of Concern after being alerted by the authors that Figure 3A was fabricated and/or falsified by the research technician who performed the experiments in the animal pulmonary physiology laboratory of Duke University (1). The experiment has been repeated, and the updated findings appear below. There was no obvious difference in airway hypersensitivity between WT and TSC1-deficient female mice and reduced airway hypersensitivity in TSC1KO male mice compared with WT male mice following methacholine challenge, in contrast with the original report. The revised experiments were completed by Barbara Theriot at Duke University.

Portions of the text related to this panel and Figure 3A are corrected as shown below.

Abstract (fourth sentence):

Here, we determined that mice lacking TSC1 exhibit a developmental block of iNKT differentiation at stage 2 and skew from a predominantly iNKT-1 population toward a predominantly iNKT-17 population.

Results (page 1686, last paragraph):

Abnormal airway inflammatory responses in TSC1KO mice. To determine whether the shift from iNKT-1 to iNKT-17 may affect iNKT cell function in vivo, we examined $\alpha$-GalCer-induced changes in airway inflammation and hyperresponsiveness. In this model, IL-17 produced by iNKT cells induces contraction of bronchial smooth muscle and neutrophil infiltration, resulting in increased airway resistance (18, 42). Twenty-four hours after $\alpha$-GalCer treatment, both TSC1KO and WT female mice had similar but weak responsiveness to aerosolized methacholine. WT male mice showed obvious dose-dependent airway responses; however, for the TSC1KO mice, the level of hyperresponsiveness was decreased compared with that of WT mice (Figure 3A). For $\alpha$-GalCer-treated mice, the total cell numbers present in the bronchoalveolar lavage (BAL) fluid from TSC1KO mice were significantly elevated as compared with those of the WT mice (Figure 3B). Neutrophils, but not macrophages, were particularly increased in TSC1KO BAL fluid (Figure 3, C and D). Moreover, following $\alpha$-GalCer administration (Figure 3E), neutrophilic infiltration in interstitial lung tissue was more apparent for TSC1KO mice than for WT mice and occurred simultaneously with upregulation of IL-17 and downregulation of Ifng mRNA levels in TSC1KO mice (Figure 3F). Similarly, challenge of TSC1KO mice with Streptococcus pneumoniae, which may contain ligands to stimulate iNKT cells $(43,44)$, induced more severe neutrophil infiltration, correlated with increased IL-17 but decreased IFN- $\gamma$ expression in iNKT cells, compared with that in WT mice (Figure 3, G and H). Taken together, these observations suggest that TSC1 deficiency results in enhanced IL-17 production and neutrophil infiltration in vivo following iNKT stimulation. However, airway hyperresponsiveness is reduced in TSC1-deficient mice. Further studies are needed to determine whether other immune cells that may affect airway responsiveness are affected in $T s c 1^{f / f l}-C d 4 C r e$ mice.

Discussion (page 1689, first paragraph):

In this report, we demonstrated that TSC1/mTOR signaling plays critical roles in iNKT cell development and effector lineage decision. We have shown that deficiency of TSC1 results in impaired iNKT terminal maturation, a reversal of the normal iNKT-1 predominance over iNKT-17, and elevated IL-17 levels and neutrophil infiltration in vivo following iNKT cell activation.

A

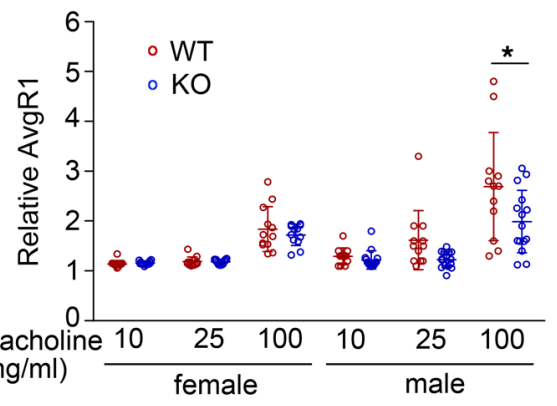

Figure 3 legend (page 1689):

Abnormal airway responsiveness, neutrophilic infiltration, and Il17a mRNA levels in the lungs of TSC1-deficient mice. WT and TSC1KO mice were treated intranasally (i.n.) with $2 \mu \mathrm{g} \alpha$-GalCer in $50 \mu \mathrm{l}$ PBS. Twenty-four hours later, changes in airway resistance to succeeding doses of aerosolized methacholine were assessed. Mice were then euthanized for collection of BAL fluid, and lung tissues were harvested for histologic examination. (A) Airway response to methacholine. AvgRT, average total pulmonary resistance. Relative AvgRT was presented as ratios to baseline AvgRT after methacholine challenge in individual mouse. Each symbol represents 1 mouse. (B) Total leukocyte numbers in BAL fluid. (C) Percentages of macrophages and neutrophils in the BAL fluid. (D) Representative H\&E staining of WT and 
TSC1KO BAL fluid infiltrates. Arrows and arrowheads represent neutrophils and macrophages, respectively. (E) Enhanced interstitial infiltration in TSC1KO lungs. Representative H\&E staining of lung thin sections is shown. (F) mRNA levels of Il17a (increased) and Ifng (decreased) in the lungs of TSC1KO mice 5 hours after $\alpha$-GalCer treatment. (G) Neutrophil numbers in the lungs after S. pneumoniae infection. Ctrl, uninfected; Infect, infected. (H) mRNA levels of indicated cytokines in iNKT cells isolated from lungs after $S$. pneumoniae infection. ${ }^{*} P<0.05 ;{ }^{* *} P<0.01 ;{ }^{* *} P<0.001,2$-way ANOVA $(\mathbf{A})$; Student's $t$ test $(\mathbf{B}, \mathbf{C}, \mathbf{F}-\mathbf{H})$. Data are representative of 2 or 3 independent experiments with 12 female WT and TSC1KO mice (A), 12 male WT and 15 male TSC1KO mice (A), 4 mice (B, C, F) and 5 mice $(\mathbf{G}$ and $\mathbf{H})$ per group in each experiment. Original magnification, $\times 400(\mathbf{D}) ; \times 200(\mathbf{E})$.

The authors regret the errors and appreciate the opportunity to correct the article.

1. JClin Invest. 2017;127(11):4216. https://doi.org/10.1172/JCI98066.

\section{Erratum}

\section{Host expression of PD-L1 determines efficacy of PD-L1 pathway blockade-mediated tumor regression}

Heng Lin, Shuang Wei, Elaine M. Hurt, Michael D. Green, Lili Zhao, Linda Vatan, Wojciech Szeliga, Ronald Herbst, Paul W. Harms, Leslie A. Fecher, Pankaj Vats, Arul M. Chinnaiyan, Christopher D. Lao, Theodore S. Lawrence, Max Wicha, Junzo Hamanishi, Masaki Mandai, Ilona Kryczek, and Weiping Zou

Original citation: J Clin Invest. 2018;128(2):805-815. https://doi.org/10.1172/JCI96113.

Citation for this erratum: J Clin Invest. 2018;128(4):1708. https://doi.org/10.1172/JCI120803.

During the preparation of this manuscript, errors were introduced into the first sentences of the Abstract and Introduction as well as the labels for Figures 2 and 3. The corrected sentences and labels are below.

Abstract, first sentence:

Programmed death-ligand 1 (PD-L1, B7-H1) and programmed cell death protein 1 (PD-1) pathway blockade is a promising therapy for treating cancer.

Introduction, first sentence:

Therapeutic blockade of programmed death-ligand 1 (PD-L1, B7-H1) or programmed cell death protein 1 (PD-1) with mAbs leads to durable tumor control in a minority of patients across many cancer histologies $(1,2)$.

Figure 2, D, E, F and I:

The mouse genotype should be $\mathrm{PD}-1^{-/}$.

Figure 2, G-I:

The dotted lines should be labeled Anti-PD-1.

Figure 3, $\mathrm{F}$ and $\mathrm{G}$ :

The labels for the $x$ axes should be ID8 TDLN.

The errors have been corrected in the HTML and PDF versions of the manuscript.

The JCI regrets the errors. 